\title{
PLASMOCYTOMA OF THE JEJUNUM: DIAGNOSIS BY DOUBLE-BALLOON ENTEROSCOPY
}

\author{
Adriana Safatle-Ribeiro, Thiago Souza, Elisa Baba, Eduardo Oppitz, Osmar Kenji, Paulo Sakai
}

\section{INTRODUCTION}

Multiple myeloma (MM) or Kahler's disease is a malignant neoplasia of the lymphoplasmocyte lineage, characterized by uncontrolled and progressive proliferation of an abnormal plasmocyte clone within the bone marrow (BM) with subsequent production of monoclonal immunoglobulin.

On the other hand, focal plasmocyte tumors (plasmocytomas) with formation of tumorous masses without medullary involvement may rarely affect the gastrointestinal tract (GIT) and are denominated solitary extramedullary plasmocytomas. The main extramedullary site of plasmocytoma is the jejunum. We report a patient who presented with jejunal plasmocytoma and emphasize the importance of the double-balloon endoscope for diagnosis of the lesion. ${ }^{1,2}$

\section{CASE REPORT}

A 72 year-old white man presented with a history of abdominal discomfort and intermittent diarrhea in addition to weight-loss (4 $\mathrm{kg}$ in 5 months) and iron deficiency anemia. Physical examination revealed the presence of a non-fixed, slightly hardened mass at the mesogastrium.

Upper digestive endoscopy and colonoscopy did evidence no alterations. Abdominal computed tomography scan revealed thickness of small intestine (Figure 1).

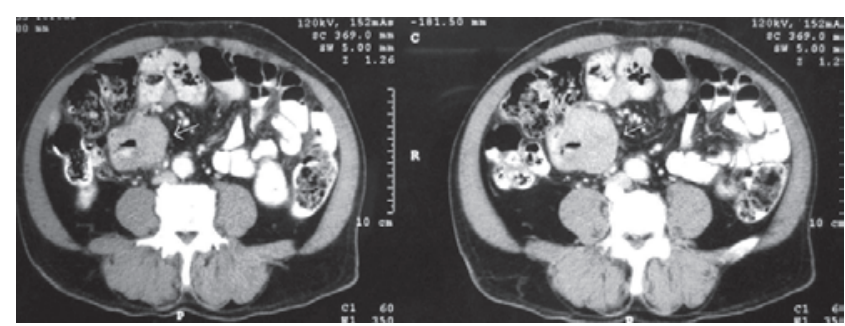

Figure 1 - Abdominal computed tomography scan evidences thickening of jejunal wall.

Department of Gastroenterology, São Paulo Univesity Medical College, São Paulo/SP, Brazil

Email: adrisafatleribeiro@terra.com.br
Double-balloon enteroscopy detected at mid jejunum an ulcerated and infiltrative lesion with imprecise limits, covered with fibrin and necrotic areas, involving $2 / 3$ of the lumen and measuring approximately $5 \mathrm{~cm}$ of extension (Figure 2).

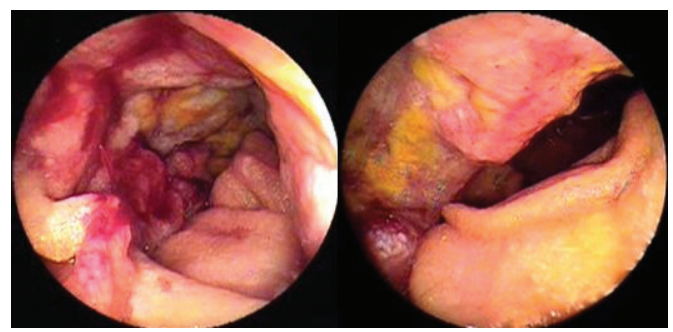

Figures $\mathbf{2 a}$ and $\mathbf{2 b}$ - Double-balloon enteroscopy reveals an ulcerated and infiltrative lesion of the jejunum.

Histopathologic examination showed enteric infiltration by plasmocytoma - Kappa chain producing multiple myeloma (Figure 3). Immunohistochemistry was positive for CD31, CD20, CD138, and Kappa with rare Lambda cells (Figure 4). Hematological evaluation did not confirm medullary and/or systemic disease, i.e. peripheral blood electrophoresis without $\mathrm{M}$ protein, negative Bence-Jones protein and aspirative bone marrow exam without abnormalities.

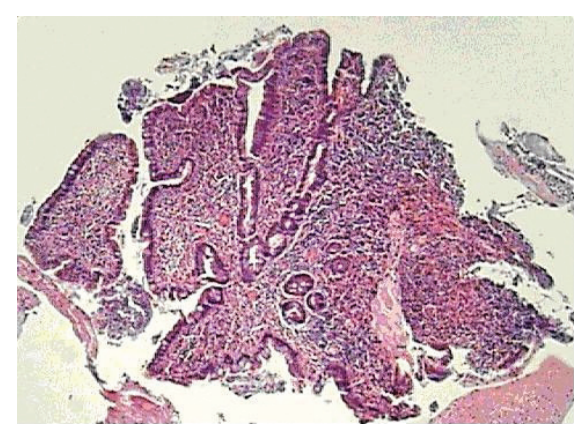

Figure 3 - Optical microscopy demonstrates massive infiltration by small malignant cells in the jejunal mucosa (HE, 40X, biopsy).

The patient underwent laparotomy, with enterectomy involving $30 \mathrm{~cm}$ of the jejunum and right hemicolectomy due to the presence of an infiltration in the wall of the as- 


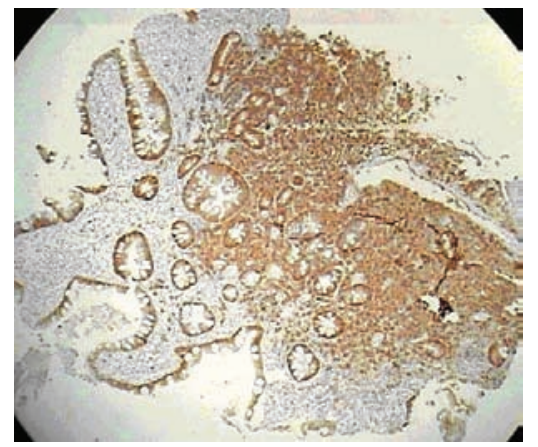

Figure 4 - Immunohistochemistry for CD138 shows positive plasmocyte cell lineage (100X, biopsy).

cending colon (Figure 5). The postoperative period was uneventful and the patient was discharged from the hospital on the $5^{\text {th }}$ postoperative day. Peritoneal gavage cytology was negative for neoplasic cells and the histological study of the surgical sample confirmed the diagnosis of plasmocytoma of the jejunum (with free resection margins), including an inflammatory infiltrate in the colonic wall (Figure 6). Thirteen resected lymph nodes were free of disease.

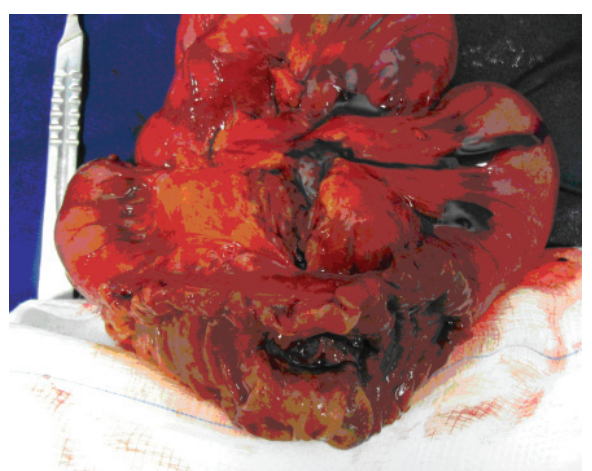

Figure 5 - Surgical specimen showing small intestinal lesion with invasion of the colon.

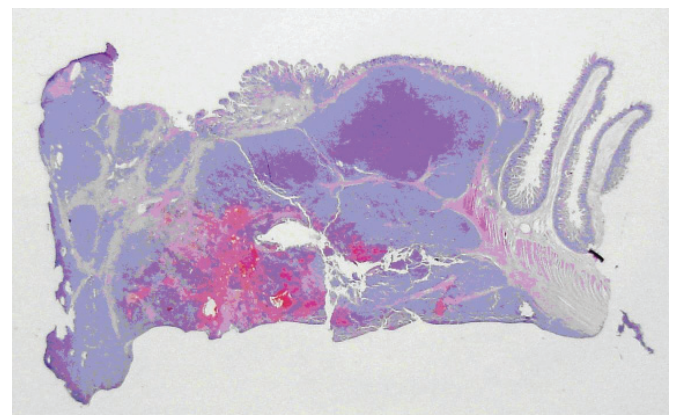

Figure 6 - Thickening of the jejunal wall caused by infiltration of the tumor (optical microscopy, HE, 10X).

\section{DISCUSSION}

MM is an unusual malignant neoplasia of plasmocyte lineage, whose clinical manifestations are based on the systemic effects of the monoclonal protein and on the concomitant humoral immunodeficiency state, as well as on the effects of bone marrow invasion by malignant cells. ${ }^{1}$

The disease affects patients with a mean age of 60 years (less than $2 \%$ of the cases occur before the age of 40 ) and typically presents with bone pain, mild anemia, fatigue, weight-loss and an elevated sedimentation rate.

Extramedullary MM involvement is more frequent than formerly supposed, affecting spleen, liver, lymph nodes and kidney in up to $65 \%$ of the cases. ${ }^{3}$

The diagnosis of plasmocytoma of the gastrointestinal tract is uncommon (12\% of the cases), and it may be seen as a primary or a secondary lesion to the systemic disease, usually with impairment of the jejunal segment.

Clinical suspicion of isolated plasmocytoma is infrequent, except for the cases with systemic disease. The commonest clinical presentation is abdominal pain, palpable mass, anemia, weight-loss, nausea and vomiting. Bleeding and intestinal obstruction may also occur. ${ }^{1,3,4,5}$

In the presence of a palpable mass, imaging tests are the first to be performed. Contrasted study may be used, and should be avoided in the presence of a sub occlusive presentation and is contraindicated in patients with intestinal obstruction. The presence of lesions in multiple segments is rare. Abdominal computed tomography scan helps to determine the size, local regional extension and impairment of other organs. However, histological diagnosis is obtained through fragments of biopsy performed through endoscopic examination or through surgical procedure. There are reports of utilization of fine needle puncture for the establishment of the diagnosis. The presence of M protein in peripheral blood helps with diagnostic complementation and may occur in cases of solitary plasmocytomas. ${ }^{1,6,7}$

Double-balloon enteroscopy allows endoscopic study and biopsies of the entire small intestine, and permits histological diagnosis throughout. Capsule endoscopy may be used; however, it should be avoided in obstructed patients, and does not have the potential for histological confirmation.

Histology demonstrates the involvement of the entire thickness of the wall, with atypical plasma cells and bizarre plasmocytes in varied levels of differentiation. In the presence of associated systemic disease, comparisons between the aspirate and tumorous histology may help the diagnostic conclusion. The immunohistochemical study is fundamental to confirm the B cell origin. 
In some cases angiographic studies have been applied, especially in patients with systemic disease, and demonstrate the presence of hypervascular lesions, positive for gallium- $67 .^{3}$

Such tumors should be distinguished from other neoplasias and correlated with clinical presentation and possible systemic disease. The differential diagnosis between intestinal lymphoma, adenocarcinoma and other rare lesions may be difficult, emphasizing the role of histological confirmation.

Treatment of MM is based on chemotherapy and the use of corticosteroids, with improvement occurring in most patients. Presently, eradication of all tumor cells can only be obtained with bone marrow transplant combined with chemotherapy. Surgical resection remains as the optimal therapeutic modality in cases of solitary extramedullary plasmocytoma, mainly if related to symptoms, with cure as a possibility when associated with chemotherapy. ${ }^{7,8,10}$

Prognosis of MM is related to the cell load. Poor prognosis is associated with a high cell load, reflected as anemia, reduced renal function, hypercalcemia, extensive bone impairment and high peaks of monoclonal protein. ${ }^{8,9,10,11}$

In summary, the diagnosis of a solitary plasmocytoma of the small intestine presents difficulties, considering its rarity, unspecific presentation and localization. Double-balloon enteroscopy, allowing access and performance of biopsies, enables histological diagnosis. Surgical resection remains the main therapeutic modality, but clinical and endoscopic follow-up is recommended because of the possibility of relapse or appearance of another plasmocyte tumor.

\section{REFERENCES}

1. Griffiths AP, Shepherd NA, Beddall A, Williams JG. Gastrointestinal tumor masses due to multiple myeloma: a pathological mimic of malignant lymphoma. Histopathology. 1997;31:318-23.

2. Yamamoto H, Sekine Y, Sato Y, Higashizawa T, Miyata T, Iino S, et al Total enteroscopy with a nonsurgical steerable double-balloon method. Gastrointest Endosc. 2001;53:216-20.

3. Tsuruda T, Ohashi T, Uezono S, Kato J, Nagamoto Y, Sakata J, et al. Extramedullary plasmocytoma of the jejunum. Intern Med. 1996;35:422-6.
4. Curcio CM, Feinstein RS, Humphrey RL, Jones B, Siegelman SS. Computed tomography of entero-enteric intussusception. J Comput Assist Tomogr. 1982;6:969-74.

5. Park IG, Lee YJ, Park do H, Han SY, Lee GH, Myungg SJ, et al. A case of jejunal stricture due to extramedullary plasmocytoma. Korean J Gastroenterol. 2003;42:67-71.

6. Goldstein WB, Poker N. Multiple myeloma involving the gastrointestinal tract. Gastroenterology. 1966;51:87-93. 
7. Okamura S, Tsujigami K, Muguruma N, Ito S, Wakatsuki S, Sano T, et al. Jejunal extramedullary plasmocytoma. Gastrointestinal Endoscopy. 2005;61:107-8.

8. Homma K, Ihzumi T, Nemoto K, Ohnishi Y. Primary extramedullary plasmocytoma of the small intestine. Int J Hematol. 1992;56:179-84.

9. Rygaard-Olsen, Boedker A, Emus HC, Olsen HA. Extramedullary plasmocytoma of the small intestine: a case report studied with electron microscopy and immunoperoxidase technique. Cancer. 1982;50:5736.
10. Merle H, Cassuto JP, Laselve L, Blein L, Delmont J. Malignant plasmocytoma of the duodeno-jejunal angle. Apropos of a case. Chirugie. 1976;102:678-82.

11. Minami A, Iwai A, Watanabe $\mathrm{Y}$, Nagamatsu H Aono S, Kato S, et al. Two cases of inflammatory bowel disease with multiple myeloma. J Gastroenterol. 1999;34:629-33. 